\title{
Just another brick in the wall: unravelling the epigenetic mechanism underlying tumour initiation of testicular germ cell cancer
}

\author{
R Buettner ${ }^{\star}, 1$ \\ ${ }^{1}$ Institute of Pathology, University Hospital Medical School Cologne and ClO Köln Bonn, Kerpenerstraße 62, D-50937 Cologne, \\ Germany
}

\begin{abstract}
Clarifying the genomic basis of human diseases and its many breakthroughs in cancer medicine has distracted our view from the importance and complexity of epigenomic information. A wall of dogmas awaiting their destruction was built: DNA methylation was proposed to occur passively but subsequently active DNA demethylation was discovered (Cedar, 1988). Histone methylation was thought to be irreversible until the first histone methylase, lysine-specific demethylase 1, was discovered and shown to provide essential cues for transcriptional regulation (Shi et al, 2004; Metzger et al, 2005). Subsequently, understanding the epigenome and its key writer enzymes, methylases and demethylases, provided important insights into pathomechanisms of aging, cancer and inflammation. The current study by Kristensen et al (2014) now closes another gap by providing evidence for an epigenetic mechanism driving intratubular germ cell neoplasia unclassified (ITGCNU), the precursor lesion for invasive testicular germ cell cancer (TGCC).

The pathogenic model for TGCC is founded on the landmark description of ITGCNU, also refered as carcinoma in situ (CIS) or testicular intratubular neoplasia (TIN) by Skakkebaek (1972) solely on morphological observations. The model hypothesised ITGCNU cells as equivalents of differentiation-arrested primordial germ cells (PGCs), which provide a basis for further progression into invasive TGCCs. Hence, the mechanism of differentiation arrest is the entry port into tumourigenesis and may be influenced by environmental factors underlying the rapid increase in incidence of TGCC in most Western countries.

The authors studied human adult and fetal testicular tissues and showed that PGCs undergo active demethylation during a critical and narrow time window of fetal development, involving the demethylase AID/APOBEC1 and the base excision repair machinery, MBD4, APEX1 and PARP1. Thus, after fertilisation and reprogramming of the zygote, PGCs undergo a second round of epigenetic reprogramming during germ cell maturation.
\end{abstract}

The authors then investigated the demethylation machinery in ITGCNU cells and showed that they closely parallel PCGs and maintain their genome in a demethylated and undifferentiated state, even in the presence of de novo methyltransferases. Acquisition of further somatic mutations, that is, in the stem cell factor receptor c-KIT (CD117) or other oncogenic drivers then steer expanding germ cells after puberty into further progression of TGCC (Tian et al, 1999; Biermann et al, 2007).

Where will we go from here and will the understanding of basic principles of tumourigenesis translate into better treatment of patients? It is obvious that the development of targeted cancer therapies resulted from profound understanding of oncogenic driver mutations and from subsequent application of selective inhibitors such as the tyrosine kinase inhibitor Imatinib targeting the genomically modified abl-kinase or gain-of-function mutations in c-KIT. Likewise, it will be rewarding to develop specific and selective inhibitors for epigenetic enzymes or their pathways and to determine the window of application in tumours that rely on their pathologically altered activity. Thus, there is no single breakthrough in oncology but rather many individual steps of developing personalised and selective cancer medicines.

\section{REFERENCES}

Biermann K, Göke F, Nettersheim D, Eckert D, Zhou H, Kahl P, Gashaw I, Schorle H, Büttner R (2007) c-KIT is frequently mutated in bilateral germ cell tumours and down-regulated during progression from intratubular germ cell neoplasia to seminoma. J Pathol 213(3): 311-318.

Cedar H (1988) DNA methylation and gene activity. Cell 53(1): 3-4.

Kristensen DG, Nielsen JE, Jørgensen A, Skakkebæk NE, Rajpert-De Meyts E, Almstrup K (2014) Evidence that active demethylation mechanisms maintain the genome of carcinoma in situ cells hypomethylated in the adult testis. Br J Cancer 110(3): 668-678. 
Metzger E, Wissmann M, Yin N, Müller JM, Schneider R, Peters AH, Günther T, Buettner R, Schüle R (2005) LSD1 demethylates repressive histone marks to promote androgen-receptor-dependent transcription. Nature 437(7057): 436-439.

Shi Y, Lan F, Matson C, Mulligan P, Whetstine JR, Cole PA, Casero RA, Shi Y (2004) Histone demethylation mediated by the nuclear amine oxidase homolog LSD1. Cell 119(7): 941-953.

Skakkebaek N (1972) Possible carcinoma-in-situ of the testis. Lancet 2(7776): 516-517.
Tian Q, Frierson Jr HF, Krystal GW, Moskaluk CA (1999) Activating c-kit gene mutations in human germ cell tumors. Am J Pathol 154(6): 1643-1647.

(c) (1) (2) This work is licensed under the Creative Commons (c) ${ }_{\text {BY }}$ NC SA Attribution-NonCommercial-Share Alike 3.0 Unported License. To view a copy of this license, visit http://creativecommons. org/licenses/by-nc-sa/3.0/ 